\title{
BMJ Open All-cause mortality following low-dose aspirin treatment for patients with high cardiovascular risk in remote Australian Aboriginal communities: an observational study
}

\author{
Yuejen Zhao, ${ }^{1}$ Kanakamani Jeyaraman, ${ }^{2}$ Paul Burgess, ${ }^{3}$ Christine Connors, ${ }^{4}$ \\ Steven Guthridge, ${ }^{5}$ Louise Maple-Brown, ${ }^{2,5}$ Henrik Falhammar (i) ${ }^{2,6}$
}

To cite: Zhao Y, Jeyaraman $\mathrm{K}$, Burgess $\mathrm{P}$, et al. All-cause mortality following low-dose aspirin treatment for patients with high cardiovascular risk in remote Australian Aboriginal communities: an observational study. BMJ Open 2020;10:e030034. doi:10.1136/ bmjopen-2019-030034

- Prepublication history and additional material for this paper are available online. To view these files, please visit the journal online (http://dx.doi org/10.1136/bmjopen-2019030034).

Received 24 February 2019 Revised 20 September 2019 Accepted 07 November 2019

Check for updates

(c) Author(s) (or their employer(s)) 2020. Re-use permitted under CC BY-NC. No commercial re-use. See rights and permissions. Published by BMJ.

For numbered affiliations see end of article.

Correspondence to Dr Henrik Falhammar; henrik.falhammar@ki.se

\section{ABSTRACT}

Objectives To evaluate the benefit and risk of low-dose acetylsalicylic acid (aspirin) in patients from remote Aboriginal communities in the Northern Territory, Australia. Design Retrospective cohort study using primary care and hospital data routinely used for healthcare. Aspirin users and non-users were compared before and after controlling confounders by matching. Marginal structural models (MSM) were applied to ascertain the benefit and risk. Setting The benefit and harm of aspirin were investigated in patients aged $\geq 18$ years from 54 remote Aboriginal communities.

Participants None had a previous cardiovascular event or major bleeds. Patients on anticoagulants or other antiplatelets were excluded.

Intervention Aspirin at a dose of 75-162 mg/day. Outcome measures Endpoints were all-cause, cardiovascular mortality and incidences of cardiovascular events and major bleeds.

Results 8167 predominantly Aboriginal adults were included and followed between July 2009 and June 2017 (aspirin users $n=1865$, non-users $n=6302$, mean followup 4 years with hospitalisations 6.4 per person). Univariate analysis found material differences in demographics, prevalence of chronic diseases and outcome measures between aspirin users and non-users before matching. After matching, aspirin was significantly associated with reduced all-cause mortality ( $\mathrm{HR}=0.45$ : $95 \% \mathrm{Cl} 0.34$ to $0.60 ; p<0.001)$, but not bleeding ( $H R=1.13: 95 \% \mathrm{Cl} 0.39$ to 3.26; $p=0.820$ ). After using MSMs to eliminate the effects of confounders, loss of follow-up and time dependency of treatment, aspirin was associated with reduced allcause mortality ( $\mathrm{HR}=0.60$ : $95 \% \mathrm{Cl} 0.47$ to $0.76 ; \mathrm{p}<0.001$ ), independent of age $(\mathrm{HR}=1.06 ; \mathrm{p}<0.001)$, presence of diabetes $(H R=1.42 ; p<0.001)$, hypertension $(H R=1.61$; $p<0.001)$ and alcohol abuse $(H R=1.81 ; p<0.001)$. No association between aspirin and major bleeding was found ( $\mathrm{HR}=1.14: 95 \% \mathrm{Cl} 0.48$ to $2.73 ; \mathrm{p}=0.765)$. Sensitivity analysis suggested these findings were unlikely to have been the result of unmeasured confounding.

Conclusion Aspirin was associated with reduced allcause mortality. Bleeding risk was less compared with survival benefits. Aspirin should be considered for primary

\section{Strengths and limitations of this study}

- The strength of the study is the assessment of aspirin as primary prevention in a real-world situation for mainly Aboriginal patients with high cardiovascular risk. There are no clear guidelines for this setting

- The study uses routinely collected clinical data and advanced statistical methods to correct the effects of confounding factors and loss to follow-up.

- This process is easy to use and well suited for routine monitoring and balance checking between benefit and risk related to a specific intervention.

- Although causality cannot be tested with this study design, the fact that low-dose aspirin was associated with reduced all-cause mortality without significant increased risk of bleeding brings more light on the risk-benefit assessment of aspirin.

- Generalisability of our results is limited to the remote Aboriginal population. Reliability of the results was tested for unmeasured confounding.

prevention in Aboriginal people with high cardiovascular risk.

\section{INTRODUCTION}

The Northern Territory (NT) is a large and sparsely populated area in the northern Australia, with a much greater proportion of Aboriginal people (28\%) in the population than Australia as a whole $(3 \%) .{ }^{1}$ The majority of Aboriginal people in the NT live in remote communities. Aboriginal patients are at high risks for diabetes, ischaemic heart disease (IHD) and stroke including ischaemic stroke (IS) with premature onset of these conditions-two decades earlier than Australian average. $^{2-4}$ It is well established that among people with diabetes, cardiovascular disease is the primary cause of death, occurring in people with diabetes or pre-diabetes. ${ }^{5}$ Given 
the high prevalence of diabetes (predominantly type 2 diabetes) and other cardiovascular risk factors (such as hypertension and smoking) from a young age among remote Aboriginal population, ${ }^{6} 7$ the local guidelines for remote health practitioners recommend low-dose acetylsalicylic acid (aspirin) for primary and secondary prevention of occlusive cardiovascular events. ${ }^{8}$ However, some studies have reported that aspirin can potentially increase the risk of haemorrhagic stroke (HS) and major bleeds, ${ }^{9-12}$ especially for patients older than 70 years. ${ }^{13-15}$ Thus, benefits from aspirin have not been reliably demonstrated for primary prevention and, in this setting, it remains unclear whether the benefits can fully negate the potential risks. ${ }^{16}{ }^{17}$ International and Australian guidelines recommend aspirin to be considered in patients with diabetes aged $\geq 50$ years at increased cardiovascular risk and not at increased risk of bleeding. ${ }^{18} 19$ The new US guidelines recommend aspirin use for the primary prevention in 40-70 year-olds at high cardiovascular risk but not at increased bleeding risk. ${ }^{20}$ However, the population in remote areas of NT is distinct, and there is a need to investigate whether routine use of aspirin for primary prevention is beneficial or harmful in this much younger patient population with high cardiovascular risk.

\section{METHODS}

A retrospective cohort study was designed to evaluate the risks and benefits of aspirin as primary prevention in patients with high cardiovascular risk. We analysed two NT Department of Health administrative data universes: Primary Care Information System (PCIS) and hospital morbidity (HM). The data sets record information on clinical diagnosis and management in 54 remote clinics and all five public hospitals managed by the Department of Health. Quality of demographic data was assessed as complete and reliable (eg, Aboriginal status was $99.98 \%$ complete with $98 \%$ accuracy) by independent audit. ${ }^{21}$ The PCIS data collection included information on demographics, medications and clinical problems. Demographic information included age, sex, usual place of residence and Aboriginal status. All diagnoses in the problem data set were coded using the International Classification of Primary Care version 2 (ICPC). The medication chart included the drug name, dose, start and end date. HM data were used to assess the effects of aspirin. HM data included admission date, separation date, diagnoses, separation mode and demographic information. All diagnoses in HM data were coded using the International Statistical Classification of Disease and Related Health Problems version 10 (ICD). Table 1 lists ICPC and ICD by conditions. Prevalence data on diabetes, renal failure, cancer, chronic obstructive pulmonary disease (COPD), hypertension, lipid disorder, overweight and alcohol abuse were also analysed. Both PCIS and HM data sets contained death information.

Patients who were aged 18 years and over on 1 July 2017 (date of birth before 1 July 1999, see figure 1) and had a

\begin{tabular}{|c|c|c|}
\hline Diagnosis & PCIS (ICPC code) & HM (ICD code) \\
\hline Diabetes & T89, T90 & E10-E14 \\
\hline Stroke & K90 & $\begin{array}{l}\text { I60-I62 (haemorrhagic); } \\
\text { I63 (ischaemic); I64 } \\
\text { (unspecified) }\end{array}$ \\
\hline $\begin{array}{l}\text { Transient ischaemic } \\
\text { attack }\end{array}$ & K89 & G45.9 \\
\hline Ischaemic heart disease & K75 & 121 \\
\hline $\begin{array}{l}\text { Other cardiovascular } \\
\text { events }\end{array}$ & K77-K80, K91 & $142-152,165-169$ \\
\hline $\begin{array}{l}\text { Other major bleeding } \\
\text { events }\end{array}$ & $\begin{array}{l}\text { A10, D14-D16, } \\
\text { R06, U06 }\end{array}$ & $\begin{array}{l}\text { R58, K92.1, K92.2, } \\
\text { K62.5, R04, R31 }\end{array}$ \\
\hline
\end{tabular}

HM, hospital morbidity; ICD, International Statistical Classification of Disease and Related Health Problems; ICPC, International Classification of Primary Care; PCIS, Primary Care Information System.

prescription recorded in PCIS from 1 July 2009 to 30 June 2017 were included. Patients were excluded if they (1) had previous cardiovascular disease including IHD, coronary angioplasty or coronary artery bypass grafting; or (2) had previous intracerebral haemorrhage, subarachnoid haemorrhage, gastrointestinal bleed, haematemesis, melaena or other known bleeding diathesis such as thrombocytopenia; or (3) were on anticoagulant or other antiplatelet agents.

By using the medication chart, we identified three groups: (1) patients with no recorded exposure to aspirin, (2) patients who were prescribed aspirin at a dose of 75-162 mg/day anytime during the study period, and (3) patients with occasional use of aspirin for fever or pain relief. If patients were categorised into multiple groups, group 2 took the priority, followed by group 3 and then group 1. There are no pharmacies in remote Aboriginal communities in the NT and aspirin is administered in hospital or remote clinic. Group 2 was considered aspirin users, and groups 1 and 3 were merged as non-aspirin users. Medication start date was defined as the date of first aspirin prescription for aspirin users. The date of first prescription of any other medication recorded in medication chart was considered the medication start date for non-aspirin users. Interactions between different medications were not considered in this study.

Outcomes were measured by (1) all-cause death and survival time; (2) cardiovascular-specific death; (3) incidences of IHD, IS, transient ischaemic attack (TIA) and other cardiovascular disease; and (4) incidences of HS, and other major bleeds (defined in table 1) such as gastrointestinal haemorrhage, haematemesis and melaena to analyse the risks. The incidence was defined by a new diagnosis, if it happened 6 months after the medication start date and was proven there were no same conditions (mapped ICD and ICPC codes in table 1) previously by searching HM and PCIS data. Deaths were identified by PCIS and HM. Patient records were linked and traced until the occurrence of a condition or death or both, or identified as a disease-free encounter. Cardiovascular-specific 


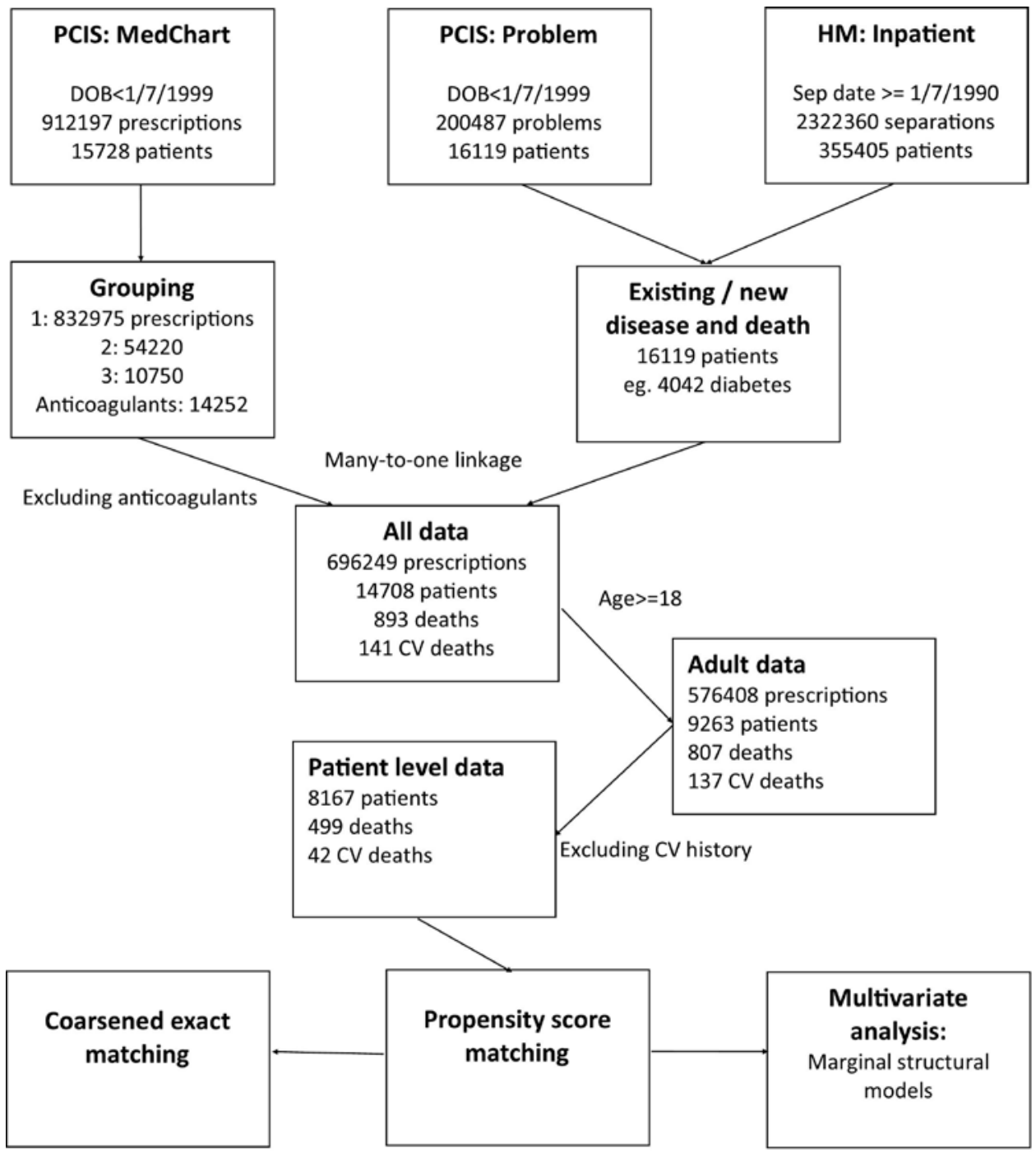

Figure 1 Illustrative flow chart of data linkage and assembly of patient cohort. CV, cardiovascular; DOB, date of birth; HM, hospital morbidity; PCIS, primary care information system; Sep, separation.

deaths were identified by a circulatory chapter ICD code as the principal diagnosis at hospital discharge (death). All stroke cases were validated manually in terms of stroke type by reviewing the medical records. Among a total of 22 undetermined cases, 4 cases were reclassified as HS, and 11 IS and 7 remained undetermined. The undetermined strokes were analysed as a separate stroke category. Follow-up time was calculated as the number of days between the medication start date and the separation date of the subsequent or recurrent admission. Follow-up time was right censored at: the latest of either presentation date of the last clinic visit or the separation date of the last admission for patients discharged alive; or for the patients without any visit or hospitalisation identifiable prior to medication start date. The survival time was measured as the length of the follow-up time. The right censoring means the actual survival time may be longer than the follow-up time.

PCIS demographics, medication chart and problem data were linked with HM by the unique hospital registration number. The flow chart in figure 1 illustrates the data linkage, grouping and calibration process. Prescriptionlevel MedChart data from PCIS were used as the base for this study. Existing and new diagnoses and health outcome measures were sourced from both PCIS and HM (all separations data collected since 1 July 1990). Many-to-one patient-level deterministic linkage was done to connect prescriptions with prevalent and incident diseases and health outcomes. Inclusion and exclusion criteria were implemented at the patient level. The full study protocol is provided as online supplementary material. Patientlevel data were analysed univariately using frequency, proportion, mean with $95 \% \mathrm{CI}$ and median with IQR as appropriate. The Kaplan-Meier survival method was used to compare incidences (disease-free survival), all-cause and cardiovascular-specific deaths between aspirin and non-aspirin group by diabetes status adjusted for loss to follow-up without controlling other confounders. Loss to follow-up and right censoring were analysed using survival and assumed missing at random (rather than assuming to be a confounder). To reduce the effect of confounding by indication, we controlled confounders by 
1:1 propensity score matching (PSM) and then coarsened exact matching (CEM), ${ }^{22}{ }^{23}$ based on 11 variables (age, sex, Aboriginal status, and diagnosis of diabetes, renal disease, cancer, COPD, hypertension, hyperlipidaemia, overweight and alcohol disorder). CEM provided a more rigorous approach to controlling confounders, which requires identical matching for multiple discrete variables and coarsened continuous variables. ${ }^{23}$

For multivariate analysis, patient-month data were created to better analyse the impacts of loss to follow-up and time-dependent confounder. A marginal structural model (MSM) was used to assess treatment effects. ${ }^{24}$ The MSM discretises patient data to take into account timevarying interventions such as start and end date of aspirin and time-dependent confounders such as age. The counterfactual outcomes under different interventions were quantified using a multivariate proportional hazards model for all-cause and disease-free survival. ${ }^{25}$ All statistical analyses were performed using Stata/SE statistical software (StataCorp, Texas, USA). Statistical significance was determined at the alpha $=0.05$ level. Sensitivity analysis was undertaken using Mantel-Haenszel bounds to test the influence of unmeasured confounders. ${ }^{26}$ Bootstrap with 2000 replicates was applied to test the robustness of MSMs. As part of the sensitivity analysis, Aboriginal peoplespecific analysis (data not shown) was also performed which did not show substantially different results, without Aboriginal status as an independent variable.

\section{Patient and public involvement}

Patients and/or members of the public were not directly and personally involved with design, data provision, analysis and publication of the study.

\section{RESULTS}

A total of 8167 patients were included (figure 1), the majority were Aboriginal peoples $(71.1 \%)$ and females $(51.4 \%)$ with a median age of 42 years (IQR 32-51). The aspirin group was 10 years older with 15.3 more hospitalisations per patient than the non-aspirin group (table 2). Table 2 compares the baseline characteristics between aspirin users and non-users. Out of the 1865 aspirin users, 1360 had diabetes (72.9\%), 5.2 times the non-users proportion $(\mathrm{p}<0.001)$. The aspirin group had longer follow-up (6.1 years vs 3.3 years), less loss to follow-up $(0.4 \%$ vs $26.0 \%)$ and more deaths compared with the non-users $(10.2 \%$ vs $4.9 \%)$. The aspirin group had higher rates of chronic disease (such as renal disease and cancer). The unadjusted HR between aspirin and non-aspirin users was 4.13 for HS ( $p=0.004)$, but 0.72 for bleeding $(\mathrm{p}=0.014)$, which was inconsistent and indicative of selection bias and loss to follow-up. Figure 2 shows Kaplan-Meier survival curves comparing aspirin users and non-users for all-cause (figure 2A,B) and cardiovascularspecific (figure 2C,D) death by diabetes status (as a proxy for cardiovascular risk), corrected for loss to follow-up. The survival curves indicate that aspirin was more likely to be associated with reduced all-cause mortality $(\mathrm{HR}=0.66$ : $95 \%$ CI 0.55 to $0.80 ; \mathrm{p}<0.001$, see table 2 ) but complicated by the follow-up time and presence of diabetes. Further stratification by diabetes status shows the preventive effect of reduced all-cause mortality was more pronounced in patients with diabetes.

PSM selected 1012 pairs of aspirin and non-aspirin users with similar characteristics (table 2). There was no statistically significant difference in the 11 potential confounders between matched aspirin users and nonusers (all $\mathrm{p}>0.15)$. Aspirin was associated with reduced all-cause death $(\mathrm{HR}=0.45$ : $95 \%$ CI 0.34 to $0.60 ; \mathrm{p}<0.001)$ and likely a lower level of complications (6.75 vs 6.85) and Charlson index (1.33 vs 1.35) in aspirin users, after accounting for potential confounders by PSM. There was no statistically significant increase in risks of major bleeding ( $\mathrm{HR}=1.02, \mathrm{p}=0.940)$ or $\mathrm{HS} \quad(\mathrm{HR}=1.23, \mathrm{p}=0.823)$ for the aspirin group.

CEM found 295 pairs of aspirin and non-aspirin users with identical characteristics and coarsened age categories (table 2). Aspirin was found to be associated with a lower level of all-cause death (HR=0.33: $95 \%$ CI 0.16 to $0.70 ; \mathrm{p}=0.003)$. There was no clear and consistent pattern in terms of changes in specific cause of death category between three methods (table 2). The protective association of aspirin with IHD (HR=0.84: 95\% CI 0.12 to 5.99; $\mathrm{p}=0.865)$ and cardiovascular death $(\mathrm{HR}=0.44: 95 \%$ CI 0.04 to $4.86 ; \mathrm{p}=0.503$ ) was non-significant. There was not enough cases to allow estimation of HR for HS, IS and TIA in the CEM selected pairs. No elevated risk in major bleeding was detected $(\mathrm{HR}=1.13, \mathrm{p}=0.820)$ for the aspirin group.

The multivariate MSMs using patient-month data indicate that aspirin was protective against all-cause death (HR=0.60: $95 \%$ CI 0.48 to 0.75 ; $\mathrm{p}<0.001)$ after taking into account both loss to follow-up and time dependency of the variables. This lower level of all-cause death appeared not to be associated with lower cardiovascular deaths. This association was also independent of age $(\mathrm{HR}=1.06$ : 95\% CI 1.05 to $1.06 ; \mathrm{p}<0.001)$ and other cardiovascular risks such as diabetes (HR=1.42: 95\% CI 1.17 to 1.71; $\mathrm{p}<0.001$ ), hypertension ( $\mathrm{HR}=1.61: 95 \%$ CI 1.31 to 1.98 ; $\mathrm{p}<0.001)$ and alcohol abuse $(\mathrm{HR}=1.81: 95 \%$ CI 1.49 to $2.19 ; \mathrm{p}<0.001)$. No association between aspirin and major bleeding was found (HR=1.14: 95\% CI 0.48 to 2.73; $\mathrm{p}=0.765$ ). Associations between aspirin use and cardiovascular death, incidences of IHD, IS as well as HS were not detected by MSMs. Sensitivity analysis indicated that the chance of overestimation (slightly greater than underestimation) of the preventative effect was small $(\mathrm{p}<0.001$, data not shown). The HR estimation was deemed robust (table 3) and unlikely influenced by hidden bias.

\section{DISCUSSION}

This observational cohort study provides empirical evidence that aspirin use for primary prevention in patients with high cardiovascular risk living in remote 


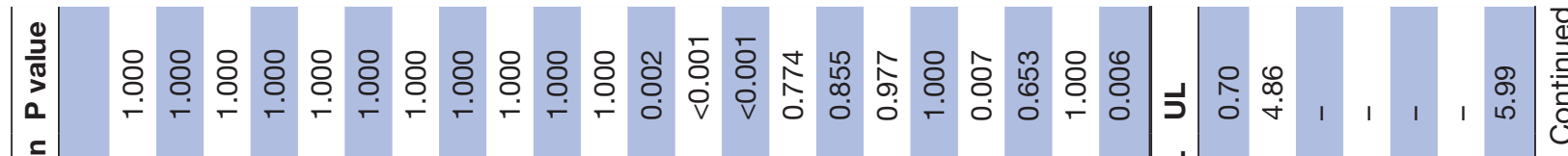

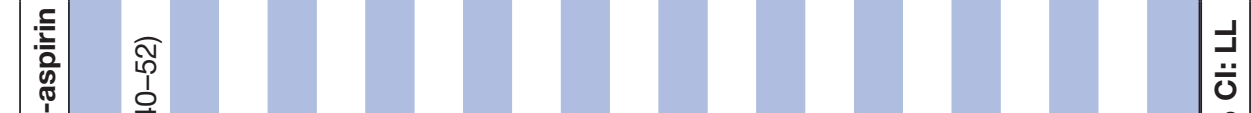

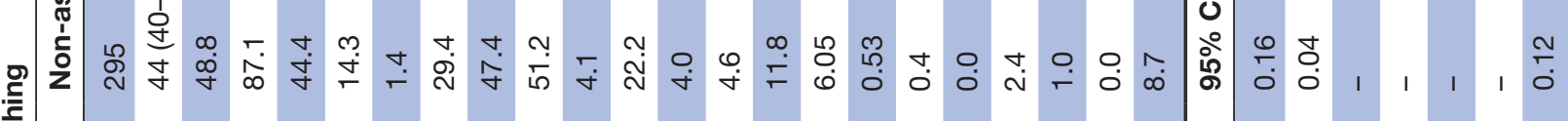

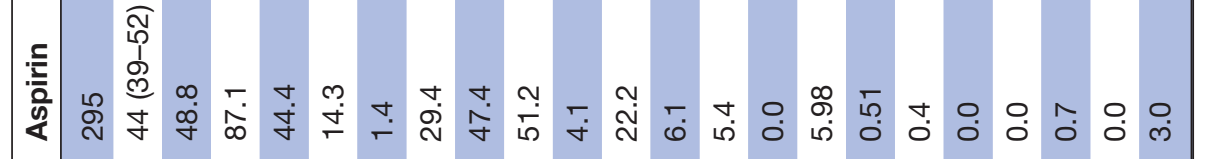

$\stackrel{0}{\frac{\pi}{3}}$

$\stackrel{8}{\infty}$

ลิ

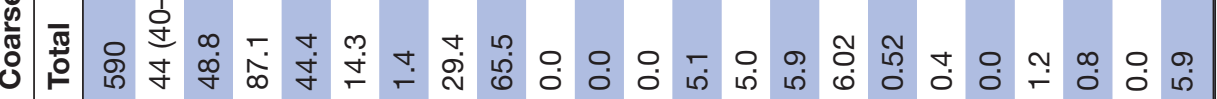

๓̣

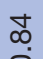

紊

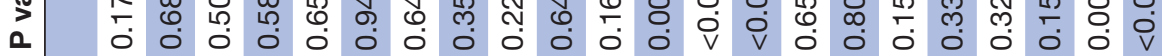

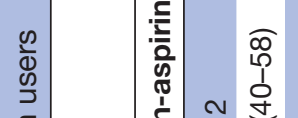

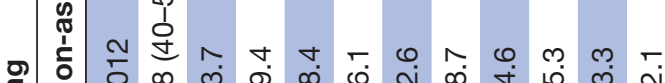

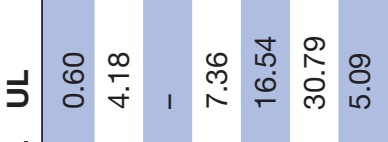

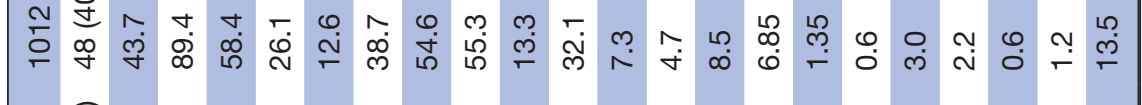
$\widehat{0}$

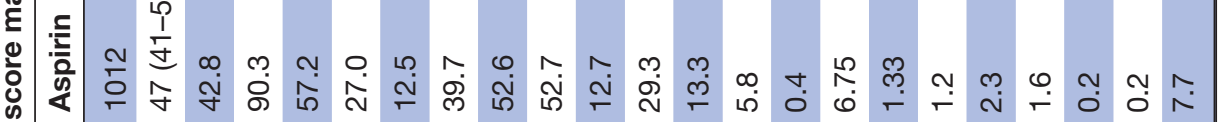

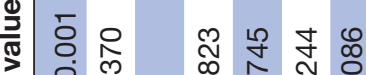

蛋

ฮ

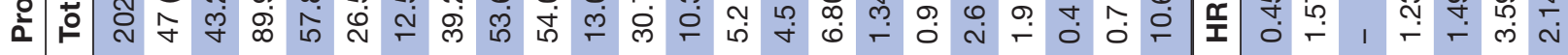

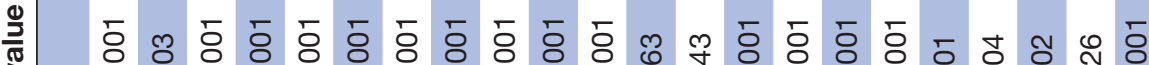

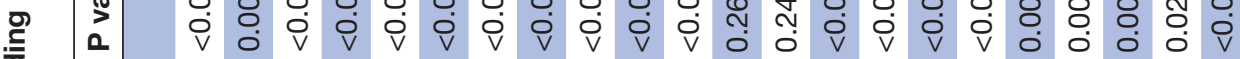

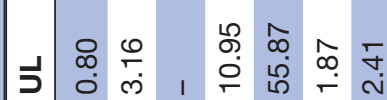

空

先

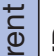

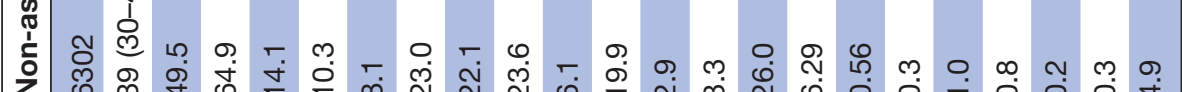

Ј

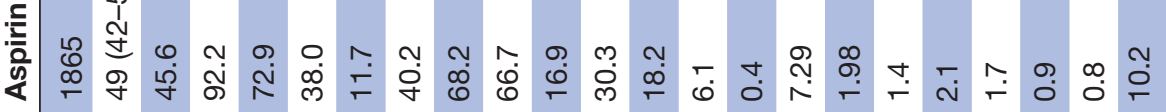

商

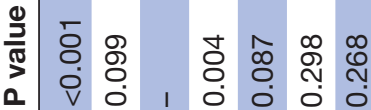
เิ

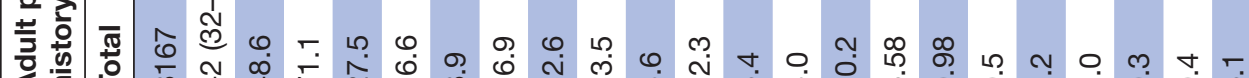

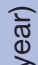

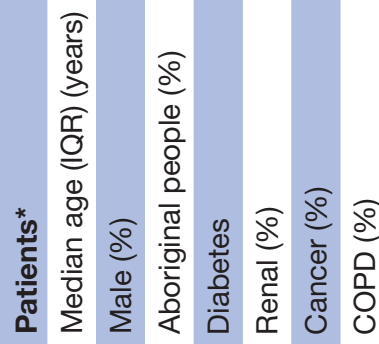

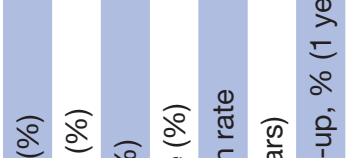

व
อ

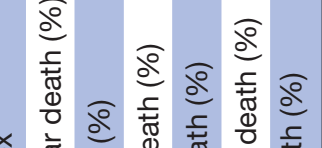

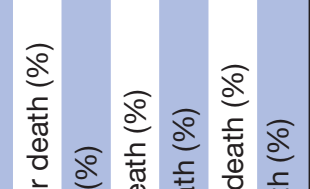

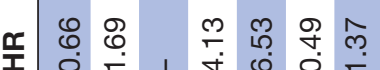

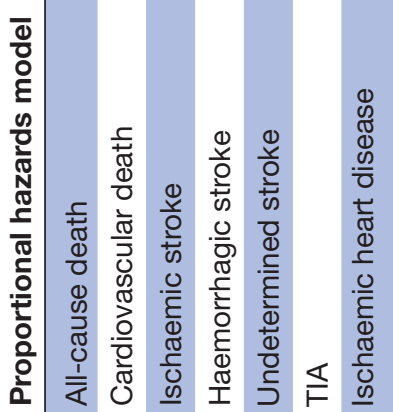


Australia was associated with significant reduction in allcause mortality. The risks of HS or other major bleeds were either undetectable or less in comparison with clear benefits of improved survival.

Several meta-analyses including the systematic review done by the US Preventive Services Task Force have indicated that aspirin reduces the risk of non-fatal myocardial infarction over 10 years. ${ }^{1627}$ This benefit begins sometime within the first 5 years of aspirin use. Our study indicated a $16 \%$ reduction in the incidence of IHD and $56 \%$ reduction in cardiovascular deaths after more rigorous matching by CEM (table 2). However, these results were not statistically significant, probably due to the low number of events and small sample after matching. Our findings on mortality benefits using MSM suggest that aspirin was strongly related to a $40 \%$ lower all-cause mortality, which differed from previous results showing only $6 \%$ or less all-cause or cardiovascular mortality. ${ }^{28}{ }^{29}$ The significant all-cause mortality benefit found in the present study at univariate level (34\%) became more pronounced (up to $67 \%$ ) when adjusted for confounders and the comparability improved. This substantial mortality benefits could be partly explained by the unique characteristics of our study population. Many of the previous trials using aspirin for primary prevention have included older patients with cardiovascular risk factors, ${ }^{16} 273031$ with the mean age being $55-71$ years. ${ }^{27}$ Our study population had a mean age of only 42 years, lived in remote communities and had a very high proportion of Aboriginal participants $(71.1 \%)$, who are well known to have earlier onset of multiple cardiovascular risk factors such as diabetes, hypertension, dyslipidaemia, albuminuria, obesity and renal disease. ${ }^{2-432}$ These differences are likely to have contributed to the greater benefit of aspirin in our study. However, in line with existing literature, ${ }^{27}$ we could not find a significant benefit of aspirin in reducing cardiovascular mortality.

The major adverse effect of aspirin is bleeding, even a dose less than $100 \mathrm{mg} /$ day has been reported to increase the risk of major gastrointestinal bleeding by $58 \%$ and possibly HS by $27 \% .{ }^{33}$ A recent Japanese study of patients with type 2 diabetes suggested that aspirin did not reduce the risk for cardiovascular events but increased the risk for gastrointestinal bleedings. ${ }^{11}$ However, age is a very strong risk predictor for major bleeding, along with male sex and several other cardiovascular risk factors. ${ }^{16} 34$ Interestingly, in our study we did not find any increased risk of HS or major bleedings. This may be explained by the much younger age with equal sex distribution of our study population compared with others. ${ }^{31}$ Moreover, we followed the guideline of aspirin treatment ${ }^{8}$ by assessing bleeding tendency before and during the treatment and excluded all patients with history of major bleedings.

There is compelling evidence demonstrating aspirin lowers the risks of cardiovascular deaths and events in patients with high risk of cardiovascular events. ${ }^{30} 3135 \mathrm{~A}$ meta-analysis of patients with type 2 diabetes but no prior cardiovascular disease showed a modest $10 \%$ reduction in 
A

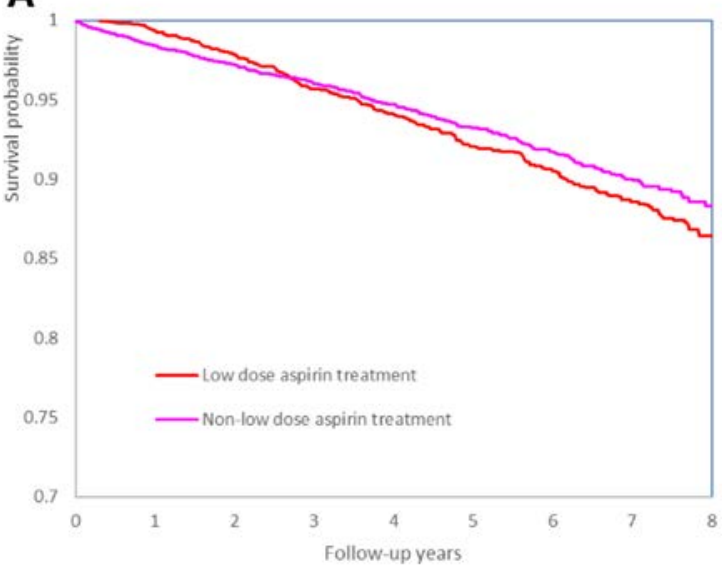

C

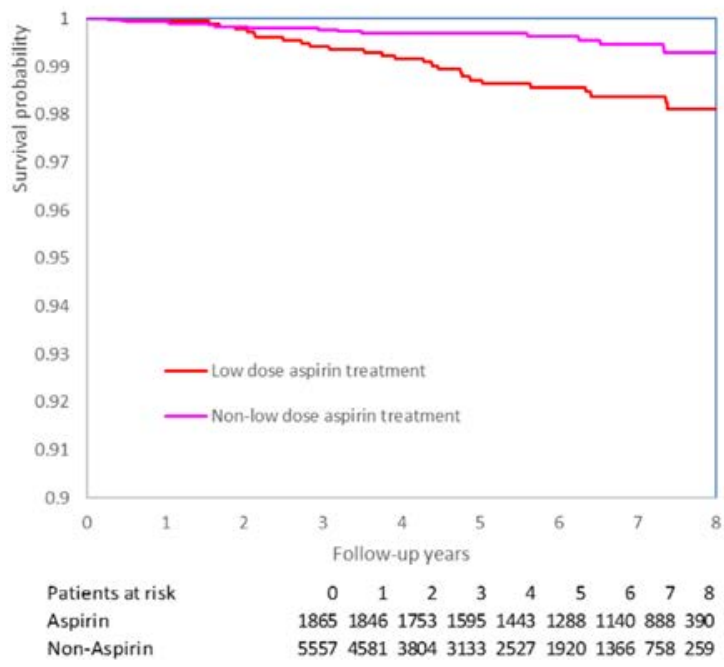

B

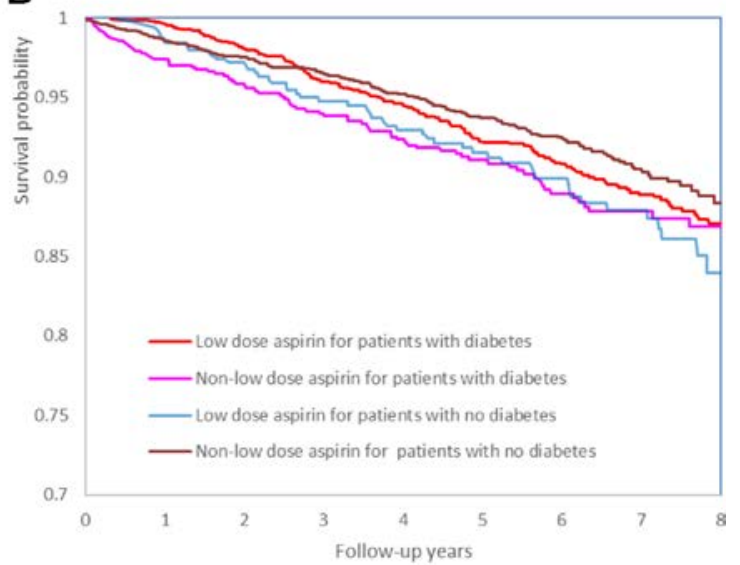

D

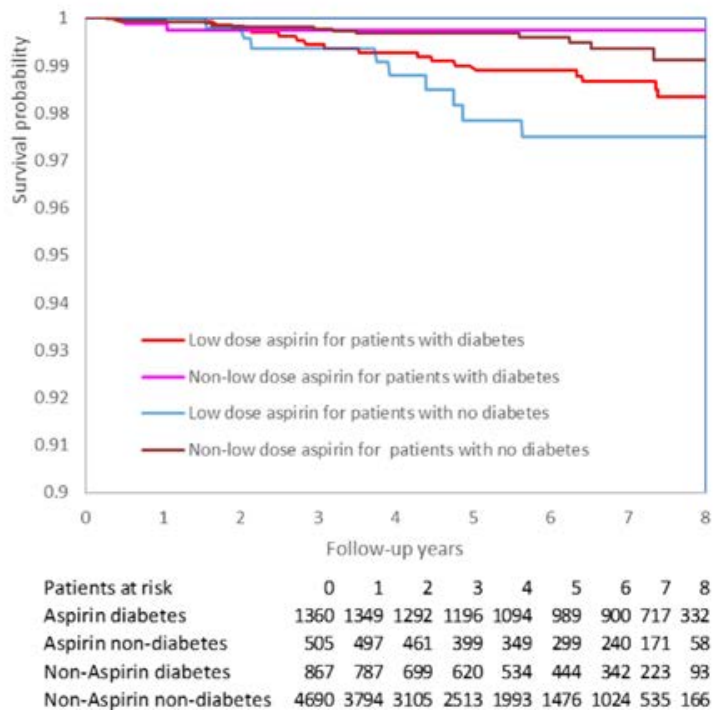

Figure 2 Kaplan-Meier survival curves by treatment groups for patients with and without diabetes. (A) All causes by aspirin group. (B) All causes by aspirin and diabetes status. (C) Cardiovascular causes by aspirin group. (D) Cardiovascular causes by aspirin and diabetes.

major cardiovascular events but no reduction in mortality. ${ }^{36}$ In a study in Australia, aspirin was found cost-effective in primary and secondary prevention. ${ }^{37}$ Moreover, in a recent randomised controlled trial of 15480 Caucasians with diabetes (mean age 63 years) but no evidence of cardiovascular disease, $100 \mathrm{mg}$ of aspirin over the follow-up of 7.4 years reduced the cardiovascular event rate by $12 \%$ but increased the risk of major bleedings by $29 \% .^{38}$ There is also conflicting evidence suggesting that aspirin has no effect on cardiovascular prevention or allcause and cardiovascular mortality, ${ }^{119-41}$ and should not be recommended for patients under 50 years of age. ${ }^{42}$ Altered platelet, endothelial and vascular smooth muscle function along with a prothrombotic proinflammatory state have been suggested for the failure of aspirin to modify the platelet response, in patients with diabetes. ${ }^{42}$

The main strength of the present study was its assessment of aspirin as primary prevention in a real-world situation of high-risk, mainly Aboriginal patients when the guidelines are unclear or controversial. We used advanced statistical methods including PSM, CEM and MSM to reduce the impacts of loss to follow-up and confounding by indication. This process may also be useful for future routine monitoring and analysis of serious adverse events related to a specific medication. The quality of data has been validated and the data linkage key has been demonstrated to be reliable. ${ }^{21}$ However, there were several limitations to our study. First, this was not a randomised clinical trial, but a retrospective observational study and cannot demonstrate true causality. Causal inference cannot be drawn as the association between treatment and outcome, which may be influenced by bias due to unknown or unmeasured confounders (such as smoking status), but CEM and MSM methodologies provided a sound approach to reveal causality by using existing observational data. ${ }^{23} 24$ Because of the higher smoking rate in Aboriginal population, confounding due to smoking is likely to lead to false-positive rather than false-negative results. Moreover, sensitivity analysis suggested there was no significant bias due to unmeasured confounding. The total level of 1-year loss to follow-up was considered acceptable $(\leq 20 \%)$ with aspirin group close to $0 \%$. Due to unavailability of 
Table 3 Marginal structural models for patient survival and bleeding risks

\begin{tabular}{|c|c|c|c|c|c|c|c|c|}
\hline \multirow{3}{*}{$\begin{array}{l} \\
\text { Aspirin } \\
\text { treatment }\end{array}$} & \multicolumn{4}{|c|}{ Benefit: reduced all-cause mortality } & \multicolumn{4}{|c|}{ Harm: bleeding after treatment } \\
\hline & \multirow{2}{*}{$\begin{array}{l}\text { HR }^{*} \\
0.60\end{array}$} & \multirow{2}{*}{$\begin{array}{l}\text { P value } \\
<0.001\end{array}$} & \multicolumn{2}{|c|}{$95 \% \mathrm{Cl}^{\dagger}$} & \multirow{2}{*}{$\frac{\text { HR }^{*}}{1.14}$} & \multirow{2}{*}{$\begin{array}{l}\text { P value } \\
0.765\end{array}$} & \multicolumn{2}{|c|}{$95 \% \mathrm{Cl}^{\dagger}$} \\
\hline & & & 0.47 & 0.76 & & & 0.48 & 2.73 \\
\hline Age & 1.06 & $<0.001$ & 1.05 & 1.06 & 1.00 & 0.905 & 0.98 & 1.03 \\
\hline Male & 1.19 & 0.053 & 1.00 & 1.43 & 2.40 & 0.005 & 1.31 & 4.40 \\
\hline COPD & 1.36 & $<0.001$ & 1.16 & 1.63 & 1.15 & 0.644 & 0.64 & 2.06 \\
\hline $\begin{array}{l}\text { Renal } \\
\text { disease }\end{array}$ & 1.19 & 0.086 & 0.97 & 1.44 & 1.46 & 0.299 & 0.72 & 2.97 \\
\hline Hypertension & 1.61 & $<0.001$ & 1.30 & 1.97 & 2.04 & 0.063 & 0.96 & 4.32 \\
\hline Overweight & 0.64 & 0.014 & 0.45 & 0.91 & 0.40 & 0.011 & 0.20 & 0.81 \\
\hline
\end{tabular}

*Bootstrap estimate.

†Bias-corrected robust estimate.

COPD, chronic obstructive pulmonary disease.

quality data, smoking was not considered to be a measurable confounder in this study. Second, our findings were limited to a population living in remote Aboriginal communities. Urban population was not included as private general practitioners data were not available. The generalisability may also be limited by the large proportion of younger Aboriginal patients, in a population with 15-21 years lower life expectancy at birth. ${ }^{43}$ Third, because the sample size was small and further reduced after PSM ( $n=2024)$ and CEM $(n=590)$, the evidence of aspirin was only robust for all-cause mortality and there were insufficient data to detect any possibly important differences in incidence of cardiovascular event between aspirin users and non-users. The results need to be interpreted cautiously due to the observational design of the study and as case numbers were often greatly reduced after matchings. Furthermore, our results were based on intention-to-treat analysis and we did not account for adherence to medications. MSM used data on start date and finish date of prescription, but there were no data on compliance, which has been reported to be low in remote Aboriginal communities (non-adherence rate for a general medication after prescription was up to $33 \%){ }^{44}$ Finally, as the mean follow-up period in our study was $<5$ years, we were unable to assess the contribution of cancer to the reduced mortality observed. ${ }^{42}$

In conclusion, aspirin as primary prevention may reduce all-cause mortality in Aboriginal patients from remote communities with high cardiovascular risk, with no related increase in risk of $\mathrm{HS}$ or major bleedings. Aspirin should be considered for primary prevention in high cardiovascular risk populations, though decision should be carefully made on a case-by-case basis.
Author affiliations

${ }^{1}$ Health Gains Planning, Department of Health, Darwin, Northern Territory, Australia ${ }^{2}$ Department of Endocrinology, Royal Darwin Hospital, Darwin, Northern Territory, Australia

${ }^{3}$ NT Medical School, Flinders University, Darwin, Northern Territory, Australia ${ }^{4}$ Top End Health Services, NT Department of Health, Darwin, Northern Territory, Australia

${ }^{5}$ Menzies School of Health Research, Darwin, Northern Territory, Australia ${ }^{6}$ Department of Molecular Medicine and Surgery, Karolinska Institutet, Stockholm, Sweden

Acknowledgements The authors thank the Northern Territory Department of Health for sponsoring this project.

Contributors YZ, KJ and HF designed the study, collected PCIS and HM data, undertook data linkage and statistical analysis and wrote the first draft of the manuscript. PB, CC, SG and LMB contributed to the literature review, methodology development, discussion and revision of the manuscript.

Funding HF was supported by the Magnus Bergvall Foundation (2017-02138 and 2018-02566), Karolinska Institutet (2016fobi49958) and the Stockholm County Council (20130614). LMB is supported by NHMRC Practitioner Fellowship (1078477).

Disclaimer The views expressed in this publication are those of the authors and do not reflect the views of the NHMRC and NT DOH.

Competing interests None declared.

Patient consent for publication Not required.

Provenance and peer review Not commissioned; externally peer reviewed.

Data availability statement Data are not available publicly.

Open access This is an open access article distributed in accordance with the Creative Commons Attribution Non Commercial (CC BY-NC 4.0) license, which permits others to distribute, remix, adapt, build upon this work noncommercially, and license their derivative works on different terms, provided the original work is properly cited, appropriate credit is given, any changes made indicated, and the use is non-commercial. See: http://creativecommons.org/ licenses/by-nc/4.0/.

ORCID iD

Henrik Falhammar http://orcid.org/0000-0002-5622-6987 


\section{REFERENCES}

1 Australian Bureau of Statistics. 2016 Census Community Profiles. Canberra: ABS, 2018. Available: http://www.censusdata.abs.gov. au/census_services/getproduct/census/2016/communityprofile/7? opendocument [Accessed 20 Dec 2018].

2 Maple-Brown LJ. The combined burden of diabetes and cardiovascular disease in Indigenous Australians. Curr Cardiovasc Risk Rep 2011;5:215-22.

3 Nguyen HD, Chitturi S, Maple-Brown LJ. Management of diabetes in Indigenous communities: lessons from the Australian Aboriginal population. Intern Med J 2016;46:1252-9.

4 Zhao Y, Guthridge S, Falhammar H, et al. Cost-Effectiveness of stroke care in Aboriginal and non-Aboriginal patients: an observational cohort study in the Northern Territory of Australia. BMJ Open 2017;7:e015033.

5 Baker Heart \& Diabetes Institute. The dark heart of type 2 diabetes. Melbourne: Baker Heart \& Diabetes Institute, 2018.

6 O'Dea K, Cunningham J, Maple-Brown L, et al. Diabetes and cardiovascular risk factors in urban Indigenous adults: results from the DRUID study. Diabetes Res Clin Pract 2008;80:483-9.

7 Maple-Brown LJ, Brimblecombe J, Connelly PW, et al. Similarities and differences in cardiometabolic risk factors among remote Aboriginal Australian and Canadian cohorts. Diabetes Res Clin Pract 2013:100:133-41.

8 Centre for Remote Health. CARPA standard treatment manual: $A$ clinic manual for primary health care practitioners in remote and Indigenous health services in central and northern Australia. 7th ed. Alice Springs: Centre for Remote Health, 2017.

9 De Berardis G, Lucisano G, D'Ettorre A, et al. Association of aspirin use with major bleeding in patients with and without diabetes. JAMA 2012;307:2286-94.

10 García Rodríguez LA, Martín-Pérez M, Hennekens $\mathrm{CH}$, et al. Bleeding risk with long-term low-dose aspirin: a systematic review of observational studies. PLoS One 2016;11:e0160046.

11 Saito Y, Okada S, Ogawa H, et al. Low-Dose aspirin for primary prevention of cardiovascular events in patients with type 2 diabetes mellitus: 10-year follow-up of a randomized controlled trial. Circulation 2017;135:659-70.

12 Gaziano JM, Brotons C, Coppolecchia R, et al. Use of aspirin to reduce risk of initial vascular events in patients at moderate risk of cardiovascular disease (ARRIVE): a randomised, double-blind, placebo-controlled trial. The Lancet 2018;392:1036-46.

13 McNeil JJ, Woods RL, Nelson MR, et al. Effect of aspirin on Disability-free survival in the healthy elderly. N Engl J Med 2018;379:1499-508.

14 McNeil JJ, Wolfe R, Woods RL, et al. Effect of aspirin on cardiovascular events and bleeding in the healthy elderly. $N$ Engl $J$ Med 2018;379:1509-18.

15 McNeil JJ, Nelson MR, Woods RL, et al. Effect of aspirin on all-cause mortality in the healthy elderly. $N$ Engl $J$ Med 2018;379:1519-28.

16 Antithrombotic Trialists' (ATT) Collaboration. Aspirin in the primary and secondary prevention of vascular disease: collaborative metaanalysis of individual participant data from randomised trials. The Lancet 2009;373:1849-60.

17 Barnett $\mathrm{H}$, Burrill P, Iheanacho I. Don't use aspirin for primary prevention of cardiovascular disease. BMJ 2010;340:c1805-2.

18 American Diabetes Association. Standards of medical care in diabetes 2018. Diabetes Care 2018;41:S3-157.

19 Royal Australian College of General Practitioners. General practice management of type 2 diabetes: 2016-18. Victoria: RACGP, 2016.

20 Arnett DK, Blumenthal RS, Albert MA, et al. ACC/AHA guideline on the primary prevention of cardiovascular disease. J Am Coll Cardiol 2019;2019.

21 Foley M, Zhao Y, Condon J. Demographic data quality assessment for Northern Territory public hospitals. 2011: Health Gains Planning, Department of Health 2012.

22 Rosenbaum PR, Rubin DB. The central role of the propensity score in observational studies for causal effects. Biometrika 1983;70:41-55.

23 lacus SM, King G, Porro G. Causal inference without balance checking: Coarsened exact matching. Polit Anal 2012;20:1-24.
24 Robins JM, Hernán Miguel Ángel, Brumback B. Marginal structural models and causal inference in epidemiology. Epidemiology 2000;11:550-60.

25 Karim ME, Gustafson P, Petkau J, et al. Marginal structural COX models for estimating the association between $\beta$-interferon exposure and disease progression in a multiple sclerosis cohort. $\mathrm{Am} \mathrm{J}$ Epidemiol 2014:180:160-71.

26 Rosenbaum PR. Observational studies. New York: Springer, 2013.

27 Guirguis-Blake JM, Evans CV, Senger CA, et al. Aspirin for the primary prevention of cardiovascular events: a systematic evidence review for the U.S. preventive services Task force. Ann Intern Med 2016;164:804-13.

28 Raju N, Sobieraj-Teague M, Hirsh J, et al. Effect of aspirin on mortality in the primary prevention of cardiovascular disease. Am J Med 2011:124:621-9.

29 Bartolucci AA, Tendera M, Howard G. Meta-Analysis of multiple primary prevention trials of cardiovascular events using aspirin. Am J Cardiol 2011;107:1796-801.

30 Okada S, Morimoto T, Ogawa H, et al. Differential effect of lowdose aspirin for primary prevention of atherosclerotic events in diabetes management: a subanalysis of the JPAD trial. Diabetes Care 2011;34:1277-83.

31 Bethel MA, Harrison P, Sourij $\mathrm{H}$, et al. Randomized controlled trial comparing impact on platelet reactivity of twice-daily with once-daily aspirin in people with type 2 diabetes. Diabet. Med. 2016;33:224-30.

32 Jeyaraman K, Berhane T, Hamilton M, et al. Mortality in patients with diabetic foot ulcer: a retrospective study of 513 cases from a single centre in the Northern Territory of Australia. BMC Endocr Disord 2019;19.

33 Whitlock EP, Burda BU, Williams SB, et al. Bleeding risks with aspirin use for primary prevention in adults: a systematic review for the U.S. preventive services Task force. Ann Intern Med 2016;164:826-35.

34 Hernández-Díaz S, García Rodríguez LA, Rodríguez LAG. Cardioprotective aspirin users and their excess risk of upper gastrointestinal complications. BMC Med 2006;4:22.

35 Roncaglioni MC, CGotPP P. Low-Dose aspirin and vitamin E in people at cardiovascular risk: a randomised trial in general practice. The Lancet 2001;357:89-95.

36 Kunutsor SK, Seidu S, Khunti K. Aspirin for primary prevention of cardiovascular and all-cause mortality events in diabetes: updated meta-analysis of randomized controlled trials. Diabet. Med. 2017;34:316-27.

37 Ademi Z, Liew D, Hollingsworth B, et al. Is it cost-effective to increase aspirin use in outpatient settings for primary or secondary prevention? simulation data from the reach registry Australian cohort. Cardiovasc Ther 2013;31:45-52.

38 The ASCEND Study Collaborative Group. Effects of aspirin for primary prevention in persons with diabetes mellitus. N Engl J Med 2018;379:1529-39.

39 Davis TME, Sillars BA, Davis WA, et al. Should aspirin be used for the primary prevention of cardiovascular disease in people with diabetes? Med J Aust 2009;191:356-7.

40 Belch J, MacCuish A, Campbell I, et al. The prevention of progression of arterial disease and diabetes (POPADAD) trial: factorial randomised placebo controlled trial of aspirin and antioxidants in patients with diabetes and asymptomatic peripheral arterial disease. BMJ 2008;337:a1840.

41 Sacco M, Pellegrini F, Roncaglioni MC, et al. Primary prevention of cardiovascular events with low-dose aspirin and vitamin E in type 2 diabetic patients: results of the primary prevention project (ppp) trial. Diabetes Care 2003;26:3264-72.

42 Bibbins-Domingo K, on behalf of the U.S. Preventive Services Task Force. Aspirin use for the primary prevention of cardiovascular disease and colorectal cancer: U.S. preventive services Task force recommendation statement. Ann Intern Med 2016;164:836-45.

43 Georges N, Guthridge SL, Li SQ, et al. Progress in closing the gap in life expectancy at birth for Aboriginal people in the Northern Territory, 1967-2012. Medical Journal of Australia 2017;207:25-30.

44 Burgess CP, Bailie RS, Connors CM, et al. Early identification and preventive care for elevated cardiovascular disease risk within a remote Australian Aboriginal primary health care service. BMC Health Serv Res 2011;11:24. 